\title{
DOS CONCEPCIONES DE LA ELUCIDACIÓN FILOSÓFICA*
}

\author{
J. ALBERTO COFFA \\ Sociedad Argentina \\ de Análisis Filosófico
}

\section{El problema}

Una buena porción de la filosofía de la ciencia contemporánea se ofrece como una serie de procesos de elucidación (en inglés, "explication"). Carnap presenta su lógica inductiva como una elucidación del concepto de creencia racional; Hempel y Salmon proponen sus modelos de explicación científica como elucidaciones de la idea de explicación; es razonable entender las múltiples teorías contemporáneas sobre la naturaleza y desarrollo del saber científico como otras tantas elucidaciones del concepto de ciencia. En el terreno propiamente científico se han caracterizado como elucidatorios una multiplicidad de procesos entre los que suele citarse la elucidación de los conceptos de Algoritmo (Church, Turing, Gödel), Dimensión (Poincaré, Menger), Consecuencia Lógica (Tarski), Verdad (Tarski), Simultaneidad (Einstein), Superficie (Riemann).

La cuestión que quisiera discutir en este trabajo es la de la naturaleza de los procesos elueidatorios. Mi propósito es identificar dos concepciones contrapuestas de esta actividad, $\mathrm{y}$ ofrecer algún indicio de un curioso hábito que exhiben buena parte de los profesionales contemporáneos de la elucidación: el sustentar al mismo tiempo ambas posturas irreconciliables, apelando a una u otra según las circunstancias lo aconsejen. Uno de mis objetivos es llamar la atención

- Este artículo es una versión modificada del trabajo expuesto en la Sociedad Argentina de Análisis Filosófico el día 11 de agosto de 1972, oportunidad en que fue discutido por Carlos Alchourrón y Tomás Simpson. 
sobre este hecho sorprendente que, pienso, merece más atención que la que ha recibido. Sería bueno entender las razones que inspiran esta conducta equívoca, pero no es $\mathrm{mi}$ intención enfrentar este difícil problema. Concluiré estas notas con algunas reflexiones casi autobiográficas acerca de la insuficiencia de una de estas dos concepciones en tanto teoria general de todo proceso elucidatorio.

\section{Elucidaciones à la Quine}

El primer modo de entender el proceso elucidatorio se halla expuesto de manera clara y coherente en la obra de Quine. En opinión de Quine, las expresiones òscuras pertenecientes a lenguajes naturales pueden agruparse en dos categorias excluyentes y exhaustivas: aquellas que merecen ser elucidadas y aquellas que deben ser evitadas. Dos de las tareas filosóficas más importantes son las de identificar aquellas expresiones que no merecen ser elucidadas y la de elucidar las restantes. Así como Carnap es el gran elucidador del pen. sar analítico contemporáneo, buena parte de la obra filosófica quineana puede verse como el intento de establecer que ciertas expresiones (de notorio predicamento en la comunidad filosófica) no merecen ser elucidadas - por ejemplo, algunas de las nociones centrales de la semántica clásica, como "significado", "analiticidad", "sinonimia", “necesidad", etc. Para Quine, las expresiones lingüísticas dignas de ser elucidadas son aquellas que aparecen en contextos que, como totalidades, son suficientemente claros y precisos como para ser de utilidad. Elucidarlas será, en esencia, reemplazarlas por otras expresiones que preserven el uso en tales contextos, afiándolo o precisándolo en todos los demás. ${ }^{1}$ Elucidar, para Quine, es eliminar.

Cualquier expresión lingüística que merezca ser elucidada debe hallarse asociada con algunos contextos que, como totalidades, posean un grado de claridad y precisión suficientes para garantizar su utilidad. El propósito de la elucida-

1 Cfr. [LPV], p. 25. 
ción es construir otra expresión que preserve el uso de la original en estos contextos favorecidos, al tiempo que refine (de modo arbitrario, pero claro) los restantes. Donde otros fundamentarían su descripción del proceso elucidatorio en la noción de significado, Quine, por razones conocidas, evita este paso, articulando su idea de elucidación alrededor de los conceptos de utilidad y uso.

Quine ilustra sus opiniones acerca de la elucidación analizando con cierto detenimiento el que es, en su opinión, uno de sus casos paradigmáticos: la definición de "par ordenado" de Wiener y Kuratowski. "Esta definición", dice Quine,

es paradigmática de lo que típicamente tratamos de hacer cuando, en actitud filosófica, ofrécemos un 'análisis' o 'elucidación' de ideas o expresiones formuladas hasta en. tonces de modo inadecuado. No pretendemos sinonimia. No pretendemos aclarar y explicitar lo que los usuarios de la expresión oscura tenían in mente desde un principio. No exponemos significados ocultos, como las palabras .'análisis' y 'elucidación' podrian sugerir: Fijamos primero las funciones peculiares de la expresión oscura que la hacen digna de preocupación, y diseñamos luego un sustituto claro y enmarcado en términos de nuestro agrado que cum. pla aquellas funciones. Más allá de estas condiciones de acuerdo parcial, dictadas por nuestros intereses y propósitos, todo otro rasgo del explicatum entra en la categoría de los 'no me importa' ([W\&O], p. 258).

Y acerca del análisis de Wiener y Kuratowski, agrega lo siguiente:

En el principio era la noción de par ordenado, defectuosa y enigmática, pero servicial. Luego los hombres hallaron que todo beneficio que derivaba de hablar de un par ordenado $\langle x, y\rangle$ podría también lograrse hablando acerca de $\{x,\{y, \phi\}\}$... Una concepción similar puede adoptarse ante todo proceso de elucidación. Elucidar es eliminar. Tenemos, para empezar, una expresión o forma de expre- 
sión que es, en algún sentido, molesta ... Pero también satisface ciertos propósitos que no queremos abandonar. Entonces encontramos un modo de satisfacer esos mismos propósitos a travéa de otros canales, usando otras formas de expresión menos molestas. Las viejas perplejidades quedan resueltas ([W\&O], p. 260).

Pero, ¿es posible "resolver perplejidades" mediante un procedimiento cuyo objetivo fundamental parece ser dejarlas de lado? La idea que Quine posee de lo que es "resolver una perplejidad" se aclara al atender sus quejas contra la actitud de los filósofos lingüistas hacia la variedad quineana de elucidación. Dice Quine:

Es irónico que aquellos filósofos sobre los que Wittgenstein ejerció su mayor influencia son los mismos que deploran más enérgicamente las elucidaciones que acabo de enumerar [se refiere al análisis russelliano de las descrip. ciones definidas, al análisis de la idea de implicación mediante la implicación material y a la elucidación fregeana de la cuantificación]. En firme defensa del hombre. común, censuran a estas elucidaciones por su distanciamiento del uso ordinario; no aprecian que es precisamente al mostrar que las partes problemáticas del uso ordinario pueden ser evitadas que mostramos el carácter meramente verbal de tales problemas ([W\&O], p. 261).

La ironía de este hecho radica, según Quine, en que de acuerdo al propio Wittgenstein:

la tarea de la filosofía no es resolver problemas sino disolverlos mostrando que no había en realidad problema alguno donde se pensaba que lo había. Esta doctrina tiene sus limitaciones pero se ajusta perfectamente a lo que es la elucidación. Porque cuando la elucidación prohibe un problema, lo hace mostrando que en un sentido importante el problema es irreal; en el sentido de que procede de usos inútiles ([W\&O], p. 260). 
Ahora bien, pareciera que no es suficiente hallar un modo de evitar el uso de proposiciones problemáticas para concluir que ellas no expresan un problema real. Toda porción problemática de un lenguaje puede evitarse, por ejemplo, cayendo en eterno mutismo o distracción. Pero ignorar un problema no es establecer su inexistencia a menos que el modo en que logremos evadirlo preserve todo lo que importa preservar. Por cierto, si es posible establecer que un uso lin. güístico problemático es innecesario, deberá admitirse que el supuesto problema asociado con él era un seudoproblema. Pero sin una petición de principio no es posible sostener -como lo hace Quine - que la mera presentación de una elucidación según su estilo establece el carácter innecesario del uso lingüístico cuestionado. Filosofar no es imitar al avestruz.

¿Hasta qué punto -en opinión de Quine- la elucidación así entendida podrá auxiliarnos en nuestro esfuerzo por en. tender, aclarar y resolver problemas filosóficos? Los escasos y oblicuos párrafos que Quine dedica a esta cuestión dificultan una respuesta categórica. Veamos, sin embargo, la breve ilustración que Quine ofrece del uso de las elucidaciones fuera del orbe de la matemática.

El tema elegido es la célebre tesis de la identidad psicofísica o, en terminología quineana, el "fisicalismo". Esta tesis, al menos en su formulación habitual, es la respuesta afirmativa al tradicional problema de si todos los estados y procesos mentales son (idénticos a) ciertos estados y proce; sos físicos. Es ésta una de las tesis centrales del materialismo clásico que ofrecía, de este modo, un principio de elucidación de los conceptos mentales. En efecto, hay aquí, para Quine, una elucidación. Pero en manos de la elusiva prosa quineana la tesis materialista clásica cobra un aspecto novedoso. Prestemos atención a sus palabras:

$¿$ Es el fiscalismo un rechazo de los objetos mentales o una teoría acerca de ellos? Ha de entendérselo como un repudio del estado de dolor o ira en favor de su concomitante 
físico; o ha de entendérselo como identificando el estado mental con un estado del organismo físico... ? Es esta última versión la que presenta un aspecto menos drástico. Hasta el lenguaje ordinario, en sus distribuciones más espontáneas, se encuadra sin violencia en el marco de este fisicalismo moderado: decimos 'Juan está dolorido', 'Juan está enojado', del mismo objeto del que decimos 'Juan es alto'. Lo que es posible decir ante todo al caracterizar a este fisicalismo moderado es que no proclama la existen: cia de diferencias categoriales insalvables entre lo mental y lo físico. Por ello algunos hallarían satisfacción al recordar que la distinción entre fisicalismo eliminativo y fisicalismo elucidatorio no es real ([W\&O], p. 256).

Con un pase de prestidigitación semántica Quine ha convertido la tesis de que lo mental o espiritual se reduce a lo físico en Ia tesis de que nada de importancia (pero, ¿para quién?) se pierde si dejamos de hablar de lo mental. Como mago de tercera que soy, apenas si me atrevo a aventurar una cautelosa conjetura acerca de lo que ha acontecido en la cita precedente: el conflicto anunciado en la primera frase entre el fisicalismo como teoría de los estados mentales y el fisicalismo como decisión de no pensar más en ellos (fisicalismo avestruz), se identifican, en la última, con el conflicto entre fisicalismo elucidatorio y fisicalismo eliminativo. Agréguese ahora la tesis de que elucidación es eliminación y el conflicto quedará disuelto; los extremos, unidos. De aquí en más podremos expresar en lenguaje elucidatorio nuestras convicciones eliminativas; podremos hablar como si el materialismo fuera una teoría (por tanto, interesante) al enunciar lo que en realidad es el producto de convencio. nes y decisiones (por tanto, irrebatible).

\section{Elucidaciones à la Tarski-Kreisel}

La segunda concepción del proceso elucidatorio aparece con 
perfiles más definidos en la obra de dos lógicos contemporáneos: Tarski y Kreisel.

Damos primero la palabra a Tarski, transcribiendo algunos de los pasajes en que describe la naturaleza de sus elucidaciones. El texto que sigue hace referencia al esfuerzo de Tarski por elucidar el concepto de definibilidad (aplicado, en particular, a la definición de clases de números):

El problema planteado en este artículo pertenece, en principio, al tipo de problema que aparece con frecuencia en el curso de investigaciones matemáticas. Nuestro interés se dirige a un término del cual podemos dar una caracterización con un contenido intuitivo más o menos preciso, pero cuya significación no ha sido establecida rigurosamente hasta el momento en matemáticas. Tratamos de construir entonces una definición de este término que, al tiempo que satisface los requisitos del rigor metodológico, expresa de modo adecuado y preciso el significado real del término. Fue un problema de esta naturaleza el que los geómetras resolvieron por primera vez cuando establecieron el significado de términos como "movimiento", "línea", "superficie", y "dimensión" ([LSM], pp. 111-2).

Más adelante agrega:

Comenzaré entonces por presentar al lector el contenido de este término, especialmente como se lo entiende actualmente en matemáticas. Las observaciones que haré a continuación ... nos permitirá captar más fácilmente las concepciones explicadas en la sección siguiente, y, sobre todo, juzgar si tales concepciones transmiten el significado real del término ([LSM], p. 112).

Luego de presentar su caracterización formal de definibilidad, Tarski señala :

Ahora se plantea la cuestión de si la definición que acabo de construir... es adecuada. En otras palabras, si de 
hecho capta el significado corriente de la noción tal como se lo conoce intuitivamente ([LSM], pp. 128-9).

En otro lugar Tarski puntualiza que:

La definición deseada no trata de especificar el significado de una palabra familiar empleada para denotar una noción nueva. Por el contrario, trata de captar el significado real de la vieja noción. Debemos, entonces, caracterizar esta noción con precisión suficiente, previamente al proceso de elucidación, para que sea posible determinar si la definición, de hecho, cumple con su objetivo ([SCT], p. 53).

Kreisel, por su parte, ha defendido de un modo aún más enfático y consistente esta misma actitud, en el contexto de su célebre defensa de la posibilidad del "rigor informal". Nos limitamos aquí a ofrecer un par de referencias en las que se enfrenta con la línea quineana. ${ }^{2}$

En [MLPM], p. 205, Kreisel rechaza la doctrina que él denomina 'pragmatista o positivista' que:

toma en serio lo que tradicionalmente se ha llamado experiencia del mundo físico (experimento y observación), pero no lo que considera como experiencia la filosofía mentalista tradicional, a saber, apreciaciones acerca de conceptos tales como validez lógica, procedimiento mecánico, prueba elemental ... En consecuencia, no acepta la significatividad de la pregunta si ciertos axiomas (o leyes) sobre tales conceptos constituyen un análisis correcto de los mismos, y entonces se habla de reemplazar tales conceptos por otros, introducidos de modo formal, de los que se supone que son útiles o adecuados para ciertos objetivos (más o menos indeterminados) : clarificación o elu-

2 Se encontrará información detallada en [FPDLE], que constituye una respuesta de Kreisel a una conferencia leída por Quine en el Collège de France. Véase también [IRCP], y [MLPM]. 
cidación son expresiones favoritas. En lo que a mí respecta ... no creo que se haya pensado con cuidado lo que se afirma acerca de la 'adecuación' de los conceptos citados: el único objetivo evidente de la introducción de estos conceptos de modo formalmente riguroso radica en su carácter de análisis correcto de los conceptos intuitivos dados. Por ejemplo, en topología, el objetivo al introducir una definición de dimensión en lenguaje topológico está evi. dentemente dado por su carácter de análisis correcto de una noción intuitiva de dimensión.

Nuestra segunda cita será la respuesta de Kreisel a la siguiente pregunta planteada por Bar-Hillel:

Nos ayudaría a entender sus opiniones saber si su par de conceptos "rigor informal vs. rigor formal" es muy semejante a la pareja carnapiana "clarificación del explicandum vs. identificación del explicatum". Tal caracterización sería de suma importancia si tengo razón al creer que es su intención aplicar su pareja de conceptos no sólo al dominio de la filosofía de las matemáticas sino también al de la filosofía de la ciencia en general ([PPM], p. 172).

La respuesta de Kreisel fue la siguiente:

Primero, en rigor, la identificación no es válida porque Carnap, sin duda alguna, niega la posibilidad de prueba o rigor formal, él no aceptaría como problema el de hallar el explicatum correcto y probarlo; por el contrario habla de reemplazar el explicandum precientífico por un expli. catum 'adecuado'.. . Segundo, es dable preguntarse si la teoría carnapiana del análisis filosófico se ajusta a su práctica; en particular, si al enfrentarse, por ejemplo, con un problema sobre inferencias inductivas no trata al explicandum como una noción inteligible con precisión y capaz de un único análisis determinado (quizás después de trazar algunas distinciones preliminares como las que fueron necesarias en el caso de la noción de conjunto). 
De modo que uno queda con la impresión que una cierta teoría acerca de la naturaleza de las cuestiones filosóficas le impide decir que está identificando una definición correcta del explicandum ([PPM], p. 176).

En términos generales, la interpretación del proceso elu. cidatorio que las citas precedentes ilustran puede caracterizarse del modo siguiente: en un proceso de elucidación es posible identificar tres componentes esenciales: primero el explicandum, un concepto definido de manera más o menos vaga que funciona como objeto de la elucidación; segundo, una clase de condiciones o requisitos derivados por medio de un análisis parcial del explicandum, condiciones que un concepto debe satisfacer si ha de servir como explicatum del explicandum dado; $y$ tercero, el explicatum, un atributo definido de manera precisa y que satisface las condiciones identificadas en el segundo paso.

Para este modo de ver las cosas, un proceso de elucidación consiste en la identificación de cada uno de estos tres elementos en el orden dado. Para quien entienda los procesos elucidatorios de este modo (emplearé la expresión "el tarskiano" para referirme a él), es posible identificar ciertos conceptos, es decir, los significados de ciertas expresiones, que son objeto de interés filosófico o matemático; y es posible (siendo fiel a su significado) clarificarlos por medio de expresiones más precisas. La relación entre el concepto original y el que lo reemplaza es, en esta concepción, simple: la expresión que describe a este último ofrece una clarificación de la asociada con el primero. Desde la perspectiva tarskiana entendemos mejor lo que es un algoritmo después de la elucidación de Church que antes de ella; entendemos mejor lo que es una dimensión después de la elucidación de Poincaré y Menger que antes de ella. No es simplemente que se haya reemplazado un concepto de algoritmo o dimensión por otro relacionado con la vieja noción de modo difícil de explicar: hemos clarificado, rigorizado y quizás extendido el explicandum. 
Es importante notar que bajo esta concepción del proceso elucidatorio el primer paso, la identificación del explican$d u m$, no es posible de error, pero el segundo sí lo es. El primer paso es simplemente la identificación del objeto de atención del elucidador y como tal no puede contener afirmaciones acerca de un objeto previamente identificado. El status metodológico de este primer estadio se asemeja al de una definición más que al de una afirmación. Por el contrario, el status de la identificación contenida en el segundo paso es más bien el de una aseveración que el de una definición. En las condiciones incorporadas a este segundo ingrediente de las elucidaciones, nos estamos dirigiendo a un objeto seleccionado en el primer estadio del proceso elucidatorio y estamos diciendo algo — verdadero o falso- de él. De este modo, si bien no tiene sentido plantear cuestiones de verdad o falsedad respecto del primer estadio de las elucidaciones entendidas de este modo, lo mismo no puede decirse acerca del segundo estadio. Aquí se hacen afirmaciones acerca de un objeto previamente identificado y tales afirmaciones serán fácticamente verdaderas o falsas, al menos en algunos casos. A pesar de que el elucidador no podría haber errado en la identificación incorporada en el primer estadio, podría, en cambio, haber errado en la identificación de los elementos del segundo, pues podría haber ofrecido condiciones que no son en verdad satisfechas por el objeto de su investigación.

Distinguiendo dos estadios donde el quineano sólo ve uno, las elucidaciones tarskianas son criticables en base a su inadecuación a la noción que se intenta elucidar. En las elucidaciones à la Quine no hay un objeto común de referencia para las expresiones empleadas antes y después de la elucidación. Esta es la razón por la que era difícil entender en qué sentido las elucidaciones à la Quine pueden resolver o disolver problemas, en vez de desplazarlos.

Se habrá observado que donde el quineano hablaba de expresiones lingüísticas y su uso, el tarskiano habla de conceptos y significados.: Por ello, el tarskiano debe soportar el 
peso - para el quineano, aplastante - de una grave respon. sabilidad, la de ofrecernos una teoría de la naturaleza de esos conceptos o significados y de sus condiciones de identidad. Hay quienes creen que esto sólo es.posible introduciendo un dominio de entidades que existan fuera del orden de la Naturaleza y es por ello que ven en la referencia a conceptos un índice inequívoco de adhesión a una semántica en la que los significados se interpretan como entidades asociadas con expresiones lingüísticas. Si se agrega a esto que los más enérgicos defensores de la línea tarskiana suelen participar de esta variedad del "platonismo", quizá se entienda un motivo por el que la sensibilidad analítica contemporánea se encuentra poco dispuesta a abrazar explícitamente la línea tarskiana.

Es este el yugo más gravoso del tarskianismo. Porque si no hubiera forma de evitar la idea errónea del significado que reverbera, por ejemplo, en algunos de los textos (citados más abajo) en que Carnap se presenta como un tarskiano, habría que concluir que el tarskianismo es incapaz de dar a sus buenas intenciones un fundamento semántico adecuado. Felizmente, no parece haber motivo para extraer esta conclusión. La obra de Sellars parece indicar que es posible desarrollar una teoría del concepto basada en el rechazo de una concepción "cosista" del significado.

\section{Ambigüedades}

Si bien, en teoría, pocas concepciones parecerán más disímiles que las que acabamos de esquematizar, en la práctica se da la curiosa circunstancia de que casi nunca es fácil decidir si un filósofo pertenece a uno u otro de los grupos mencionados. Strawson, que critica a Carnap por ignorar los problemas filosóficos en elucidaciones al estilo de Quine, ${ }^{3}$ critica también a Salmon por no disolver (à la WittgensteinQuine) el problema de la inducción.* Por su parte, Quine

3 Véase la referencia (y la respuesta de Carnap) más abajo.

- En "On Justifying Induction", Philosophical Studies, 1958, p. 30. 
critica a los filósofos oxonienses por plantear la cuestión de la significatividad de una expresión como si se tratara de una cuestión de orden fáctico, ${ }^{5}$ y Grover Maxwell observará que el gravísimo error de la semántica quineana es el de plantear la cuestión de la significatividad de una expresión como si se tratara de una cuestión de orden fáctico. ${ }^{\circ}$

Sospecho que todos tienen razón (y que están equivoca. dos), porque sospecho - según señalaba al comienzo- que en más de un filósofo contemporáneo hay al menos una acti. tud ambigua hacia la naturaleza de los procesos elucidatorios.

Con el objeto de ilustrar mi convicción al respecto, habré de concentrarme en un único ejemplo, el suministrado por la obra de Rudolf Carnap. Quizá sea él, decía, el más distin. guido elucidador de nuestro siglo; curiosamente, es en su obra donde se da, más claramente que en ningún otro lugar, la ambigüedad a que he hecho referencia.

\section{4a. Carnap, el tarskiano}

Son múltiples las elucidaciones carnapianas que parecen surgir como el producto de tareas inspiradas por la concepción tarskiana. Véase, por ejemplo, su elucidación del concepto de Verdad Lógica en Meaning and Necessity. Allí comienza Carnap por identificar su explicàndum como una noción que ha sido objeto de atención filosófica al menos desde Aristóteles, y que Leibnitz tratara de elucidar de modo no muy distinto al que sugerirá Carnap. Luego como resultado de un análisis de esta noción, Carnap enuncia una condición que no es aún una definición de verdad lógica sino "una formulación informal de una condición que toda definición de verdad lógica que se proponga debe satisfacer si ha de ser una elucidación adecuada del explicandum". Finalmente

s En su contribución a la discusión sobre su trabajo "Le Mythe de la Sig. nification", en La Philosophie Analytique, Cahiers de Royaumont, pp. 170-1.

- En la primera página de su articulo "The A Priori and the A Posteriori", en Feigl y Maxwell (Eds.), Scientific Explanation, Space and Time, U. of Minnesota Press, 1962. 
dará un explicatum del que mostrará que satisface el criterio de adecuación.

O considérese la elucidación carnapiana del concepto de Apoyo Inductivo o Creencia Racional. Una vez más, Carnap comienza por identificar el explicandum de modo informal, dejando en claro que es su intención dirigirse a un problema filosófico clásico, y no la de describir un nuevo problema con vieja terminología. El explicandum es bautizado con el término "probabilidad", observando que:

el concepto de probabilidad ${ }_{1}$ como explicandum es alucidado interpretándolo como apoyo fáctico y como cuociente de apuestas justo y como estimación de la frecuencia relativa ([LFP], p. 161).

La lógica inductiva será el desarrollo teórico de un explicatum para este explicandum. Como resultado de un análisis del explicandum (cuya naturaleza Carnap no describe) se prestan criterios de adecuación para elucidaciones aceptables

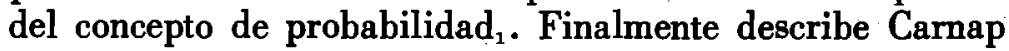
la clase de elucidaciones posibles, la que coincide con la clase de funciones de confirmación regulares, mostrando que son ellas las que satisfacen las condiciones identificadas en el segundo estadio del proceso de elucidación, y que, dice Carnap, "parecen incluir todas las funciones que podrían ser consideradas como explicata adecuados del concepto de probabilidad $_{1}$ ".

La impresión de que todo este modo de entender las elucidaciones de Carnap es el correcto se ve reforzada por diversas observaciones que Carnap ofrece en torno a la naturaleza del proceso elucidatorio. Al menos en dos ocasiones Carnap acentúa la semejanza entre su idea de elucidación y el concepto de análisis tal como lo entendía Kant. En cierta ocasión, al explicar el motivo que lo había conducido a seleccionar la terminología técnica que hemos venido empleando en este trabajo, Carnap puntualiza:

Kant llamaba a un juicio "explicativo" o "elucidatorio" 
si el predicado se obtiene por análisis del sujeto. Husserl al hablar acerca de la síntesis de la identificación entre un sentido confuso e inarticulado y otro articulado y claro, llama a este último "Explicat" del primero ... Lo que yo entiendo por explicatum y explicandum es en alguna medida semejante a lo que Langford ha llamado analisandum y analisans ([LFP], p. 3).

También en este contexto merece recordarse la respuesta de Carnap a la acusación de Strawson según la cual las elucidaciones carnapianas no pueden en modo alguno ser útiles para la solución de problemas filosóficos; y agregaba Strawson que quienes eluciden al modo de Carnap han de creer "que las cuestiones y perplejidades filosóficas no pueden tomarse con seriedad".

En su enérgico rechazo de estas acusaciones, Carnap toma como modelo de elucidación la que Frege diera de los números naturales, y ofrece de ella la siguiente interpretación:

Durante miles de años muchas personas emplearon estas palabras [se refiere a los numerales " 1 ", " 2 ", etc.], adecuadamente en lo que hace a los propósitos prácticos, y durante muchos siglos los matemáticos han tenido una teoría sistemática que contenía estas palabras. Pero aun en este caso faltaba completa claridad. Antes de Frege, nadie podía dar una caracterización exacta de los significados de estas palabras en términos no aritméticos. Por medio de la elucidación de Frege de las expresiones nu. méricas -en mi opinión uno de los mayores logros filosóficos del siglo XIx - por primera vez se aclaró la conexión lógica entre estas palabras y las partículas lógicas como "hay", "no", "o" e "idéntico". Por lo tanto, debemos decir que a pesar de su habilidad para usar expresiones numéricas, la gente en general, y aún los matemáticos antes de Frege no estaban en claro acerca del significado de tales expresiones ([RSE], p. 935).

Un último argumento en apoyo de la tesis que Carnap con- 
cebía los procesos elucidatorios a la manera Tarski-Kreisel lo ofrece su conducta en lógica inductiva, al ofrecer una interpretación de los "criterios de adecuación" de su explicandum (o, "convenciones de adecuación", como Carnap las denominaba, exhibiendo una característica ambigüedad de conducta frente a la naturaleza de estas oraciones). El tema al que quisiera referirme brevemente es su famosa defensa de tales "convenciones" contra los embates popperianos.

De haber creído que tales criterios o convenciones definen parcial e implícitamente el explicandum a elucidar, de haberlos tomado seriamente como convenciones ( $\mathrm{y}$ no como afirmaciones acerca de un objeto conceptual identificado de modo independiente de tales criterios), Carnap habría contado con una respuesta simple y directa a las críticas popperianas: le habría bastado con señalar que ellas se fundaban en una simple confusión acerca de la naturaleza de estos "axiomas". En la medida en que se los entienda como "convenciones", o como definiciones "implícitas" de su tema (al modo sugerido por el formalismo Hilbertiano) es tan absurdo cuestionar la verdad de estas convenciones como lo sería el cuestionar la adecuación de los axiomas de Peano al dominio de los números naturales cuando éste se interpreta como uno cualquiera de los dominios definidos implícitamente por tales axiomas. Lejos de optar por tal respuesta, Carnap decidió defender sus "convenciones" - elevadas finalmente al rango de axiomas de la lógica inductiva - apelando a lo que él llamó "intuición inductiva". Independientemente de la posible falta de adecuación de esta defensa, lo que es significativo en este contexto es que Carnap, tras señalar que el problema de la inducción es "la cuestión de qué clase de razones pueden darse para aceptar los axiomas de la lógica inductiva", dijo de ellas: "las razones son a priori". Y luego agregó: "Las razones están basadas en nuestro juicio intuitivo acerca de la naturaleza de la validez inductiva ([RSE], p. 978).

En síntesis, pareciera claro que hay una serie de circunstancias en las que Carnap trata los diversos explicanda que 
son objeto de su atención a la manera de Tarski-Kreisel. En particular parece ser factible en estos casos que los expli. canda son nociones identificadas antes de ofrecerse las condiciones de adecuación (convenciones, axiomas), ya que se interpreta a estas últimas como afirmaciones que no son ni verdades lógicas ni meras convenciones.

\section{4b. Carnap, el quineano}

En un pasaje de [W\&O], p. 259, Quine sostiene que su modo de entender los procesos elucidatorios coinciden con el de Carnap. A pesar de lo dicho en la sección precedente debe admitirse que es también posible construir una defensa relativamente convincente de esta tesis.

Hay a lo largo de la filosofía de Carnap una corriente persistente que se presta a la interpretación dada por Quine. Nos referimos aquí a la porción de la filosofía carnapiana que inspira su principio de tolerancia, sus distinciones de marcos lingüísticos y sus distinciones entre cuestiones externas e internas. Es este el lado del pensamiento carnapiano que en tantas ocasiones lo llevó a entender como propuestas lo que otros querían entender como afirmaciones o tesis acerca de ciertos temas.

Esta tendencia (presente ya en el $A$ ufbau y aún en su tesis doctoral Der Raum) aparece claramente en La Sintaxis Lógica del Lenguaje, donde Carnap argumenta que la polémica entre intuicionistas y antiintuicionistas no debe entenderse como expresión de opiniones divergentes sobre un mismo tema sino como la enunciación de dos propuestas (plenamente compatibles) para investigar dos marcos lingüísticos distintos.

Carnap no restringió esta "tolerancia" al dominio matemático. El secular debate entre "realistas" y "fenomenalistas" no expresa, para él, un conflicto de opiniones. La creen. cia en tal conflicto es el producto de la misma confusión entre convenciones y afirmaciones que inspira - según élbuena parte del debate entre los intuicionistas y sus adver. 
sarios. Desde el $A$ ufbau hasta sus obras tardías, fenomena. lismo y realismo, en la medida en que no son meras confu. siones, son dos propuestas alternativas y compatibles para el uso de formas lingüísticas distintas. La antigua máxima que requería una distinción frente a toda contradicción al. canza su apoteosis filosófica en el principio de tolerancia carnapiano, el que -en ocasiones extremas - parece privarnos de la posibilidad del desacuerdo filosófico fuera del terreno de las actitudes.

El ejemplo más claro del costado quineano de Carnap lo ofrece su tratamiento de los conceptos fregeanos de sentido y denotación. Dice Carnap:

Parece que Frege, al introducir la distinción entre nominatum y sentido, tenía la intención de precisar cierta distinción que había adoptado diversas formas en la lógica tradicional. Por ello, la tarea que se propuso fue una elucidación ... Los explicata elegidos fueron los conceptos de nominatum y sentido ([M\&N], p. 126).

En cuanto a los explicanda fregeanos, Carnap conjeturó (contra Church; véase [M\&N], pp. 126-7) que Frege estaba tratando de elucidar el mismo par de conceptos que Mill trató de capturar con su distinción entre denotación y connotación. En el proceso de elucidar tales explicanda, Frege formula como "condiciones de adecuación" sus célebres principios de la relación nominativa.

Hasta este punto podría parecer que nos encontramos ante un típico caso de elucidación à la Tarski: primero se identifican los explicanda; luego, en virtud de un análisis de los mismos se identifican condiciones que ellos satisfacen y que todo explicatum adecuado ha de verificar; finalmente surge el explicatum. Sin embargo, Carnap rechaza de modo categórico este modo de entender las cosas:

Los principios fregeanos, es decir, los principios de la relación nominativa no son afirmaciones que puedan ponerse en duda o refugiarse. Deben entenderse como parte de 
la caracterización de los dos conceptos y por tanto valen para ellos con fuerza analítica. Si alguien dijera - como no es mi caso- que no está de acuerdo con los principios fregeanos, en realidad estaría diciendo que entiende las palabras 'nominatum' y 'sentido' en un modo distinto al de Frege; en otras palabras, que usa conceptos distintos, y no habria, en verdad, desacuerdo ( $[\mathrm{M} \& N]$, p. 128; el subrayado me pertenece).

Póngase en conjunción la tesis de que nominatum y sentido son los explicata de Frege con la afirmación que acabo de subrayar, y lo que obtenemos por resultado es la postura de Quine. Dado que no parece haber razón alguna para asignar a la elucidación fregeana un tratamiento diferencial, parecería razonable inferir de estos textos que Carnap piensa que las convenciones de adecuación de un explicatum definen implícitamente - si bien de modo parcial- el correspondiente explicandum; y que por ello las elucidaciones no pueden errar, salvo por motivos de orden lógico.

Este es el principio de tolerancia en acción. Tras su atractivo nombre se oculta el hecho menos atractivo que, según él, tan sólo puede haber entre filósofos un desacuerdo de actitudes. Según el principio, en la medida en que los filósofos hablen de lo mismo, sus afirmaciones deben ser mutuamente consistentes; y en la medida en que ellas sean incompatibles estarán refiriéndose a temas distintos. El desacuerdo filosófico no es posible por cuanto éste supondría un objeto común de referencia, y el principio de tolerancia en realidad indica que, en el orden de lo filosófico, toda concepción coherentemente desarrollada ha de entenderse como definición implícita de su tema.

5. Naturaleza y Permanencia de los Problemas Filosóficos

Hasta aquí me he limitado a ofrecer un tratamiento casi puramente descriptivo de las dos posturas vigentes en torno a la naturaleza de los procesos elucidatorios y de una cierta ambi. güedad en su ejercicio. Permítaseme concluir estas notas con 
un voto cauteloso a favor de la línea Tarski-Kreisel. Lo que sigue es, más que un argumento, un credo.

El motivo que me aparta de la línea quineana es que sospecho que, a pesar de todo, hay algunos problemas que persisten a lo largo de procesos explicativos, que hay preguntas que - bajo formas diversas- se conservan a lo largo de la historia, preguntas y problemas cuya formulación se refina sin sufrir alteración esencial y que - suerte y genio mediante- reciben, al cabo, respuesta adecuada.

Wittgenstein, por ejemplo, creía lo contrario. Él pensaba que era un grosero error creer que la "solución" del problema de la trisección del ángulo en el siglo xIX solucionaba un problema planteado en Grecia y discutido por geómetras a través de los siglos. No había, para él, un problema de la trisección del ángulo que permaneciera en esencia inmutado a lo largo de los siglos; y aplicaba la misma perspectiva a todas las circunstancias en que los matemáticos hablaban de "problemas" no resueltos. Sin duda habría considerado absurdo pensar que el teorema de Church de 1936 da respuesta negativa a la propuesta leibniziana sobre la calculabilidad del conocimiento, o que el teorema de completidad de Gödel soluciona el problema de la suficiencia deductiva del cálculo fregeano. En Wittgenstein los únicos problemas son los que cuentan con solución presente y automática. Porque algo es un problema sólo cuando es posible buscar su solución; y para Wittgenstein sólo es posible buscar algo (por ejemplo, la solución de un problema) cuando nos es dado de modo explícito un método que identifica el modo de acceso a lo buscado. Proponer un "problema" matemático (o físico) - sin disponer de un método efectivo para su solución- es como ofrecer la primera parte de una variación sobre un tema musical y proponer que se escriba la segunda parte en el mismo estilo: en ambos casos la "búsqueda" no puede tener por objeto el descubrimiento sino la invención."

Si no fuera ya tarde, sería yo el primero en conceder a

7 Véace [RFM], p. 167; también Parte II, Sección V de [PG]. 
Wittgenstein, a Kuhn, a Quine y a tantos otros, que filósofos y científicos a menudo han confundido el planteo y solución de un nuevo problema con la solución de otro clásico, que quedaba enterrado para siempre en las arcas de la historia. Y sería también el primero en conceder que si bien, en ocasiones, el reemplazo de problemas es un mero recurso sofístico (como en la "solución" popperiana al problema de la inducción), en otras el reemplazo conceptual es condición esencial para el progreso intelectual (daría como ejemplo, en Física, el tránsito a la teoría de la relatividad, y en Matemática, el cambio de concepción acerca de la naturaleza de la geometría en el siglo xxx). Es por ello que poco costará convencerme de que amplios sectores del conocimento humano se han desarrollado en virtud de procesos que responden aproximadamente a las características que Wittgenstein y luego Quine han subrayado. Sin embargo, el monadismo intelectual extremo, la postulación del discontinuismo en la historia de las ideas - patente en Wittgenstein y latente en otros- me parece un error.

Creo, por ejemplo, que múltiples problemas asociados con el concepto de efectividad se conservan, en esencia, cuando se los formula en términos de la función recursiva. Creo que múltiples problemas asociados con el concepto de dimensión se conservan, en esencia, cuando se los formula empleando la noción topológica que Poincaré y otros describieron. Creo, además, que es posible ofrecer una teoría de la ciencia que sea un análisis correcto de la naturaleza de esa actividad y no una propuesta acerca del uso de la palabra "ciencia". Creo que las filosofías de la ciencia son, en buena medida, verdaderas o falsas.

Creo, en suma, en la posibilidad de la discrepancia filosófica y en la continuidad de buena parte de la historia de las ideas. $\mathrm{Y}$ es porque no veo de qué modo pueda preservar estas creencias desde la perspectiva quineana que ofrezco al tarskianismo mi voto irreversible (por ahora). 


\section{Conclusión}

He tratado de identificar dos modos incompatibles de entender ciertos procesos intelectuales. Sería un error creer que el conflicto es meramente verbal, reduciéndolo a un debate acerca de los procesos históricamente dados que merezcan el nombre de elucidaciones. El conflicto entre quineanos y tarskianos es real ya que el quineano sostiene que no hay proceso alguno que se desarrolle del modo que el tarskiano cree.

Podrá concederse, como yo lo hago, que hay una multitud de procesos que algunos tarskianos consideran elucidaciones (en su sentido) pero que sólo lo son en algún sentido más cercano al de Quine. Podrá concederse, como yo lo hago, que hay tarskianos que no aprecian la magnitud del orbe de lo convencional ("¿Será verdad que la ballena no es un pez?"), pero el tarskiano tiene razón al pensar que ciertos momentos esenciales de la historia del pensamiento sólo pueden entenderse desde la perspectiva que él nos ofrece. En todo caso, parece difícil construir, fuera de esta perspectiva, un esquema de nuestra historia intelectual que evite representarla como un interminable diálogo entre sordos. 


\section{BIBLIOGRAFIA}

Bar Hillel, Y.

[PPM]. "Obtaining Axioms by Reflection", en Lakatos, I. (Ed.), PPM, p. 172.

Carnap, $\mathbf{R}$.

[LFP]. Logical Foundations of Probability, University of Chicago Press, 1967.

[M\&N]. Meaning and Necessity, University of Chicago Press, 1947.

[RSE]. "Replies and Systematic Expositions", en Schilpp, P. (Ed.), PRC, pp. 859-1013.

Kreisel, G.

[FPDLE]. "The Formalist-Positivist Doctrine of Mathematical Precision in the Light of Experience", L'Age de la Science, vol. III, $\mathbf{n}^{9}$ 1, pp. 17-46.

[MLPM]. "Mathematical Logic: What has it Done for the Philo. sophy of Mathematics?", en Schoenman, R. (Ed.), BRPC, pp. 201-272.

[IRCP]. "Informal Rigour and Completeness Proofs", en Lakatos, I. (Ed.), PPM, pp. 138-171 y pp. 175-184.

Lakatos, I. (Ed.)

[PPM]. Problems in the Philosophy of Mathematics, North Holland, 1967.

Quine, W. 0.

[W\&O]. Word and Object, The M.I.T. Press, 1960.

[LPV]. From a Logical Point of View, Cambridge, 1953.

Schilpp, P. (Ed.)

[PRC]. The Philosophy of Rudolf Carnap, Open Court, 1963.

Tarski, A.

[LSM]. Logic, Semantics and Metamathematics, Oxford University Press, 1956.

Schoenman, R. (Ed.)

[BRPC]. Bertrand Russell, Philosopher of the Century, N. Y.

Wittgenstein, L.

[RFM]. Remarks on the Foundations of Mathematics, Blackwell, 1967.

[PG]. Philosophical Grammar, California, 1974. 
The aim of this paper is to describe two different views on the nature of explication, and to draw attention to the fact that philosophers sometimes accept both of these conceptions even though they are mutually incónsistent.

The first conception of explication is clearly and consistently developed by Quine. For him, the objects of explications are expressons that appear in some contexts that, as wholes, are clear and precise enough to be useful. To explicate such expressions is to identify other linguistic forms to our liking that preserve the useful usages and ascribe definite but arbitrary truth-values to all remaining sentences in which the explicated expression occurs. The Wiener-Kuratowski explication of "ordered pair" is offered as a paradigm case of explication. The only context clear and precise enough to be useful which the expression "the ordered pair $x, y$ " (or " $\langle x, y\rangle$ ") appears is

$$
\text { If }\langle x, y\rangle=\langle z, w\rangle \text { then } x=z \& y=w .
$$

The Wiener-Kuratowski definition makes this context provably true (within set theory) and ascribes definite truth-values to all other grammatical contexts in which the expression " $\langle x, y\rangle$ " occurs.

The appropriateness of this conception of explication is questioned, particularly in connection with the question whether explications in this sense can help solve or dissolve philosophical problems. Although the strategy described by Quine is appropriate to ignore problems formulated in terms of the explicandum, nothing in the explicatory operation, as described by Quine, seems to contribute to the dissolution of problems relating to the explicandum.

Another problem arising in connection with Quine's conception of explication relates to the ambiguity of the crucial appeal to usefulness. The context "to explicate an expression is to replace it by another one that preserves all contexts that, as wholes, are clear and precise enough to be useful" is, nearly enough, the only one in which the expression "explication" appears in Quine's writings. And if this context, as a whole, is not clear and precise enough to be useful then the question arises, what would an explication of "explication", as conceived by Quine, look like?

The second conception of explication has been defended by Tarski and Kreisel among others. According to this way of looking at things, 
one must distinguish the following ingredients in an explication process: (i) the explicandum or concept that is to be explicated; (ii) a set of conditions that are identified by means of an analysis of the explicandum (these are conditions satisfied by the explicandum and they ought to be satisfied by any acceptable explicatum) ; (iii) the explicatum or clearly defined concept satisfying the conditions identified in (ii). The identification of the item in stage (ii) is not to be understood as a proposal or convention but (sometimes, at least) as a factual (though not necessarily empirical) claim.

Under this, construal explications can be right or wrong, true or false. They will be wrong, for example, if they incorrectly ascribe to the explicandum features that do not correspond to it, or if they fail to ascribe to the explicandum features that are essential to it. Explications in this sense are arguably useful for the solution of philosophical problems. For example, when understood as above, it would become reasonable to say that Church's explication of effectiveness provided the link necessary to interpret his undecidability result as a solution of sorts the Leibnizian problem of the decidability of all knowledge.

To hold a Quinean conception of explication is to hold that no intellectual process is accurately described by the Tarskian understanding of explication, the main reason for this contention being that the Tarskian idea of explication relies on philosophical notions (meaning, concept, etc.) that do not resist careful scrutiny.

In spite of the incompatibility between these two conceptions, explicators often associate themselves with both of these views. This claim is illustrated by an examination of Carnap's explicatory activities. It is argued that in some contexts (in the field of semantics and also in connection with the tolerance principle) Carnap espoused a Quinean conception of explications, whereas in others (induction. and also in connection with the concepts of number and logical truth) he seemed to be relying on the Tarskian version of explication.

Summary by

J. Alberto Coffa 